\title{
Generalization of the Rosen-Zener Model of Noncrossing Interactions. I. Total Cross Sections
}

T. R. Dinterman

John B. Delos

William \& Mary, jbdelos@wm.edu

Follow this and additional works at: https://scholarworks.wm.edu/aspubs

Part of the Physics Commons

\section{Recommended Citation}

Dinterman, T. R. and Delos, John B., Generalization of the Rosen-Zener Model of Noncrossing Interactions. I. Total Cross Sections (1977). Physical Review A, 15(2), 463-474. https://doi.org/10.1103/PhysRevA.15.463

This Article is brought to you for free and open access by the Arts and Sciences at W\&M ScholarWorks. It has been accepted for inclusion in Arts \& Sciences Articles by an authorized administrator of W\&M ScholarWorks. For more information, please contact scholarworks@wm.edu. 


\title{
Generalization of the Rosen-Zener model of noncrossing interactions. I. Total cross sections*
}

\author{
T. R. Dinterman and J. B. Delos \\ Department of Physics, College of William and Mary, Williamsburg, Virginia 23185
}

(Received 15 May 1975; revised manuscript received 23 June 1976)

\begin{abstract}
The formalism developed previously to study electronic transitions by the curve-crossing mechanism is used to obtain a general model for transitions which take place due to the strong coupling of curves which do not cross. This exactly solvable model is a generalization of a model developed by Rosen and Zener. The model is shown to give respectable agreement with exact calculations at low velocities, and to go to the correct resonant-charge-exchange limit at high velocities. Predictions of the model are shown to give good agreement with the measured total cross sections for charge exchange in the sytem $\mathrm{Li}^{+}+\mathrm{Na} \approx \mathrm{Li}+\mathrm{Na}^{+}$.
\end{abstract}

\section{INTRODUCTION}

According to the Massey adiabatic criterion, transitions between electronic states with energy spacing $\Delta$ are unlikely unless the time of interaction $\tau_{0}$ is short, so that $\Delta \tau_{0} \lesssim \hbar$, or equivalently, unless the nuclear velocity is high, $v \gtrsim \Delta \tilde{R} / \hbar$, where $\tilde{R}$ is the range of the interaction. Energy changes of a few eV would be impossible in lowenergy collisions were it not for the presence of special phenomena: either the spacing between the potential curves must be small or the range of the interaction must be short in order for an electronic transition to take place at low velocities. Such a situation arises, for example, if there is a crossing between two potential curves; this circumstance has been extensively studied and is now rather well understood, at least for atom-atom collisions.

Transitions between states for which there is no crossing may also take place if the coupling between the states increases sufficiently rapidly. This is believed to occur, for example, in charge exchange involving alkali metals. The purpose of this paper is to develop, solve, and apply a model for electronic excitations between curves that are strongly coupled but do not cross. The model is very general and in principle can be applied to interactions occurring at large distances, as in the alkali metals, or to degeneracies at small distances, as in the proton-hydrogen-atom system. It could also be used to study curve crossings in the classically forbidden region. In addition, the model goes to the correct limit as the velocity becomes large and as the asymptotic splitting becomes negligible; so it can be applied to resonant charge exchange (though it leads to no new results in this case).

Rosen and Zener ${ }^{1}$ were among the first to consider the description of electronic excitations in noncrossing situations; they gave the exact solution to a model having two constant potential curves coupled by an interaction $V_{12}(\tau)$, proportional to $\operatorname{sech}(a \tau)$. Much later a closely related model was suggested by Demkov ${ }^{2}$; he obtained the exact solution for a simple exponential coupling $V_{12}=\beta e^{-\gamma \tau}$, and he discussed the behavior that would result from a more general coupling. Implicit in the simple exponential form for $V_{12}$ is the assumption that the radial component of the nuclear velocity is essentially constant through the critical region; this assumption may be valid for $b \ll R_{c}$, but it makes Demkov's formula fail for $b \gtrsim R_{c}$. The Rosen-Zener form does not have this defect; so it can become the basis for a more accurate and more general treatment-which we provide in this paper. Nevertheless, Demkov's paper is important in providing an intuitive picture of charge exchange in nearly resonant collisions (small asymptotic $\left.V_{22}-V_{11}\right)$ : at large distances the electronic states are essentially atomic, at small distances they are molecular, and in a "critical region" about a point $R_{c}$ their nature changes from atomic to molecular. It is in this critical region that electronic transitions are most likely to occur.

In previous papers, ${ }^{3-5}$ we have developed a very general formulation of two-state interactions which enables us to consider a diversity of models in a unified way: in particular, we have shown that the transition probability does not depend upon all the details of the three potential matrix elements, $V_{i j}(\tau)$, but only upon the behavior of one function, $t(s)$, defined in Eq. (10) below. In the present paper (in Sec. III) this formulation is applied to noncrossing interactions $\mathrm{s}^{6-12}$; thus we obtain a very general model with a minimal set of assumptions. A summary and outline of the results of this paper follows:

(1) In Sec. II we review the formalism developed in Ref. 4(b). We emphasize again that for the twostate case the electronic transition probability and other inelastic effects do not depend upon all the details of the potential matrix $V_{i j}(R)$ and the trajectory $R(\tau)$, but only upon the one function $t(s)$ 
defined in Eq. (10). Therefore a single solvable model (such as the Rosen-Zener model) can be applied to a great variety of systems provided that the parameters in the model are related to $t(s)$ instead of to the potential matrix or the trajectory.

(2) In Sec. III A, it is shown that the model form (11) has the properties required of the physical $t(s)$; the generalized Rosen-Zener formula (13) is thereby obtained. While it has the same sech ${ }^{2}$ $\sin ^{2}$ form as the original Rosen-Zener result, the $\operatorname{sech}^{2}$ and $\sin ^{2}$ factors are not necessarily the same as those given by Rosen and Zener. The present form (13) is more general because it involves no detailed assumptions about the potential matrix elements or the trajectory, but only the one assumption (11) about the form of $t(s)$.

(3) For any given physical system, the transition probability is approximately given by (13) if the parameters $\alpha$ and $\zeta$ can be chosen so that the model $t(s)$ [Eq. (11)] is close to the actual $t(s)$ [Eq. (10)] for that system. (It must be noted that $\alpha$ and $\zeta$ will necessarily be functions of the impact parameter.) There is of course an infinity of ways of adjusting $\alpha$ and $\zeta$ to make the model (11) approximate the physical $t(s),(10)$. For example, the model (11) can be made to approximate the real system (10) by matching Taylor coefficients at the critical point $R_{c}$ where $t(s)=1$. Alternately, Taylor coefficients can be made to match at the turning point. As another possibility, the values of $t(0)$ and $S_{\infty}$ in the model can be made to agree with the corresponding values for the physical system. These three methods are discussed in detail in Sec. III B; the last one is later shown to give under further approximations a result that is essentially equivalent to the Rapp-Francis formula.

(4) In Sec. IV A, the various resulting formulas for the transition probability are compared to corresponding results of exact calculations. It is found that the most accurate results are obtained by combining the three methods. Reasonable results are also obtained from the third method by itself; the Demkov formula is shown to be less satisfactory.

(5) In Sec. IV B, we calculate total cross sections for near-resonant charge exchange. Olson, ${ }^{6}$ basing his arguments partly upon the work of Demkov, has proposed that the cross sections for many such processes lie along a universal curve. The analysis of general models like the present one (11) is useful because it permits the study of a wide range of processes by variation of a small number of parameters; hence the validity of the proposed universal curve can be tested. For this purpose, the third method (essentially the Rapp-
Francis formula) is sufficiently accurate; the accuracy of the universal curve is shown in Fig. 6 .

(6) Finally, in Sec. IV C, we use the same method to study charge exchange in the (Li-Na) ${ }^{*}$ system. ${ }^{6-8,10}$ Using the two-state approximation, the potential curves and coupling matrix element calculated by Melius and Goddard ${ }^{8}$ are transformed into the diabatic representation, and the results are discussed. The calculated total cross section is shown to be in good agreement with the experimental results and with the more accurate calculations of Melius and Goddard. (The oscillations that appear in the experimental cross section are not reproduced by this form of the generalized Rosen-Zener formula; the model could be modified to reproduce them, but it is not clear whether this would be worth the effort, and we have not made any attempt to do so.)

There have been two other recent studies of general noncrossing problems. Nakamura ${ }^{11}$ obtained a direct numerical solution to the coupled Schrödinger equations, and compared the exact results with three forms of the distorted-wave approximation and two separable approximations. The advantage of the present approach over the distorted-wave approximations is that whenever the present exactly solvable model is valid at all, it holds for arbitrary coupling strength. Finally, a closely related formula has been obtained by Crothers, ${ }^{12}$ and applied to some similar problems. We have not carried out a detailed comparison of our formulas with his, but we expect that they will have overlapping domains of validity.

\section{REVIEW OF THE FORMALISM}

If the full wave function for the electrons and nuclei is expanded in a discrete basis,

$$
\Psi(R, r)=\frac{1}{R} \sum_{n} u_{n}(R) \phi_{n}(r, R),
$$

then the $u_{n}(R)$ obey coupled equations of the form

$$
\left[\frac{1}{2 M}\left(\frac{\hbar}{i} \frac{d}{d R}+\underline{P}\right)^{2}+\underline{V}\right] u=E \underline{u}
$$

where

$$
P_{m n}=\left\langle\phi_{m}\left|\frac{\hbar}{i} \frac{d}{d R}\right| \phi_{n}\right\rangle_{\text {el ectronic }}
$$

and $V$ is the matrix of the nuclear potential energy and the electronic parts of the Hamiltonian. If only a small number of states are involved (from here on we will assume just two), then it is possible to solve Eq. (1) numerically to obtain the exact scattering amplitudes for the system. Until recently, this would have been a slow and costly approach, but there are now available more efficient numerical methods for solving these equa- 
tions. Nevertheless, it is still useful and instructive to obtain simple analytic expressions for the transition probability, and to make use of semiclassical approximations-first because they provide important insights into the nature of the collision process, and second because the interpretation of experimental data is greatly simplified if analytic formulas are available.

In earlier work, ${ }^{3}$ it has been shown that the WKB approximation reduces the full Schrödinger equation (1) to the classical trajectory equations,

$$
\begin{aligned}
& i \hbar \frac{d c_{1}}{d \tau}=V_{12} \exp \left(\frac{i}{\hbar} \int_{0}^{\tau}\left(V_{11}-V_{22}\right) d \tau^{\prime}\right) c_{2}, \\
& i \hbar \frac{d c_{2}}{d \tau}=V_{21} \exp \left(\frac{i}{\hbar} \int_{0}^{\tau}\left(V_{11}-V_{22}\right) d \tau^{\prime}\right) c_{1} .
\end{aligned}
$$

The solution to these equations can be expressed in terms of an $S$ matrix, ${ }^{4(b)}$ so that

$$
\underline{c}(\tau \rightarrow \infty)=\underline{S} \underline{c}(\tau \rightarrow-\infty) .
$$

Because of the symmetry about the turning point, $\tau=0, \underline{S}$ can be written as a product of two factors,

$$
\underline{S}=\underline{G}_{+} \underline{G}_{+}
$$

where $\underline{G}_{+}$is the matrix such that

$$
\underline{c}(\tau \rightarrow \infty)=\underline{G}_{+} \underline{c}(\tau=0) \text {. }
$$

Unitarity and the determinant of unity imply that $\mathrm{G}_{+}$can be written in the form

$$
\underline{G}_{+}=\left[\begin{array}{cc}
\left(1-Z^{2}\right)^{1 / 2} e^{i \Gamma_{1}} & -Z e^{-\Gamma_{2}} \\
Z e^{i \Gamma_{2}} & \left(1-Z^{2}\right) e^{-i \Gamma_{1}}
\end{array}\right]
$$

Here $Z$ represents the excitation probability resulting from only the outgoing part of the trajectory, and $\Gamma_{1}$ and $\Gamma_{2}$ are phases associated with the excitation process. The transition probability for the entire trajectory is

$$
P=4 Z^{2}\left(1-Z^{2}\right) \sin ^{2}\left(\Gamma_{1}+\Gamma_{2}\right) .
$$

The quantum $S$ matrix, which is used in the partial-wave summation, is given by

$$
\boldsymbol{s}_{i j}=S_{i j} \exp \left[i\left(\eta_{i}+\eta_{j}\right)\right],
$$

where $\eta_{i}$ and $\eta_{j}$ are WKB elastic scattering phase shifts for electronic states $i$ and $j$.

For the two state case, there is a very important simplification of the classical trajectory equations (2) that enables a great variety of systems to be treated by only a few models. ${ }^{4(b)}$ Defining a new independent variable

$$
s(\tau)=\int_{0}^{\tau} V_{12}\left(\tau^{\prime}\right) d \tau^{\prime} / \hbar
$$

the equations (2) take the form

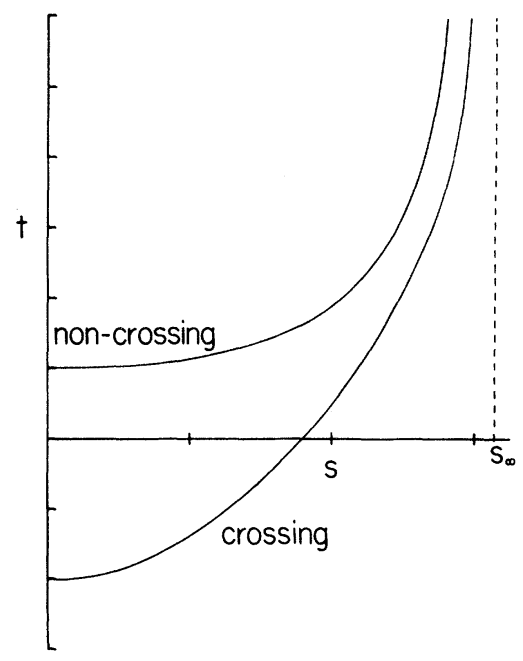

FIG. 1. $t(s)$ for a crossing and a noncrossing situation.

$$
\begin{aligned}
& i \frac{d c_{1}}{d s}=\exp \left(-2 i \int_{0}^{s} t\left(s^{\prime}\right) d s^{\prime}\right) c_{2}, \\
& i \frac{d c_{2}}{d s}=\exp \left(+2 i \int_{0}^{s} t\left(s^{\prime}\right) d s^{\prime}\right) c_{1},
\end{aligned}
$$

where

$$
t=\left(V_{22}-V_{11}\right) / 2 V_{12} \text {. }
$$

This means that all the inelastic effects contained in $\underline{S}$ depend only on the behavior of one function $t(s)$. It is easy to establish three general properties of $t(s)$ (Fig. 1): (i) At the turning point, $s=0$, $d t / d s=0$. (ii) At a crossing, $t=0$, but if there is no crossing, $t(s)$ remains of fixed sign, by convention $\geq 0$. (This assumes $V_{12}$ does not change sign.) (iii) At some finite $s=s_{\infty}, t \rightarrow \infty$, approximately as $\left(s_{\infty}-s\right)^{-p}$ with $1 \leqslant p \leqslant 2$. Assuming $V_{22}-V_{11} \simeq$ constant, then $V_{12} \propto R^{-2}$ implies $p=1$, and $V_{12} \propto e^{-\lambda R}$ implies $p=2$.

\section{GENERALIZED ROSEN-ZENER MODEL}

A. General form and solution

The above discussion implies that it is only necessary to obtain an accurate approximation to $t(s)$ in order to calculate the inelastic scattering matrix $S$. Thus we seek a model for $t(s)$ having the above properties and for which the classical trajectory equations are exactly solvable. There does exist such a form, and it is

$$
t(s)=1 /(\alpha \cos \zeta s) \text {. }
$$

The parameters $\alpha$ and $\zeta$ are both positive, and it is easy to see that $t>0$ for $0 \leq s \leq \pi / 2 \zeta$; also $d t / d s$ $=0$ at $s=0$, and $t$ has a simple pole at $s_{\infty}=\pi / 2 \zeta$. One may choose these parameters so that this 
model form (11) matches as closely as possible the true $t(s)$ for the physical system under consideration. Then the calculated $S$ matrix should be a good approximation to the true $S$ matrix.

In the Appendix, it is shown that the exact solution to Eq. (9), with the model form (11) for $t(s)$, gives

$$
\begin{aligned}
& G_{11}=F\left(B_{1}+B_{2}\right), \\
& G_{21}=-i F\left(B_{1}-B_{2}\right),
\end{aligned}
$$

where

$$
\begin{aligned}
& F=(\pi / 2)^{1 / 2}\left(\frac{1}{2}\right)^{i \gamma} \Gamma\left(\frac{1}{2}+i \gamma\right), \\
& B_{1}=\left[\Gamma\left(\frac{1}{2}(a+1-c)\right) \Gamma\left(\frac{1}{2}(-a+2-c)\right)\right]^{-1}, \\
& B_{2}=\left[\Gamma\left(\frac{1}{2}(a+2-c)\right) \Gamma\left(\frac{1}{2}(-a+1-c)\right]^{-1},\right. \\
& a=1 / \zeta, \quad c=\frac{1}{2}-i \gamma, \quad \gamma=1 / \alpha \zeta .
\end{aligned}
$$

Although the formulas are complicated, they can be evaluated numerically without difficulty.

The overall transition probability has a much simpler form (A11):

$$
P=\operatorname{sech}^{2}(\pi / \alpha \zeta) \sin ^{2}(\pi / \zeta) .
$$

This has the same form as the result obtained by Rosen and Zener, ${ }^{1}$ but the present derivation is more general, because it makes assumptions only about $t(s)$ and not about the three potentials $V_{i j}(t)$. As a consequence, the parameters $\alpha$ and $\zeta$ may have different values and a different interpretation than the parameters appearing in the Rosen-Zener paper.

As we have shown elsewhere, ${ }^{4(\mathrm{~b})}$ it is possible to describe the result of the inelastic process using only the $S$ matrix, which contains $P$ and a single associated phase. However, the $G$ matrix, although complicated, is also useful, because it provides scattering angles associated with the two trajectories taken by the system (for excitation on the incoming or outgoing part of the path).

\section{B. Specific forms for applications}

The above results are exact, if $t(s)$ is given by (11). To apply the model to a specific physical situation, it is necessary to approximate the true $t(s)$ by the model form (11); i.e., . we need a systematic way of choosing the parameters $\alpha$ and $\zeta$ to approximate the actual physical system as closely as possible. There is an infinity of ways to do this, of which we will consider three.

The model form for $t(s),(\alpha \cos \zeta s)^{-1}$, and the actual $t(s)$ for the physical system will never be identical; therefore we need to know where they should be in closest agreement with each other. Now, when $t$ is slowly varying and large $\left(2 V_{12} \ll V_{22}-V_{11}\right)$, the system may be described by elastic propagation on the diabatic potentials, and the inelastic effects are negligible. When $t$ is slowly varying and small $\left(2 V_{12} \gg V_{22}-V_{11}\right)$, the system may be described by elastic propagation on the adiabatic potentials, and again inelastic effects are negligible. Inelastic transitions take place primarily when $t$ is moderate $\left(2 V_{12} \sim V_{22}-V_{11}\right)$ and rapidly changing. Accordingly, the model should attempt to match the physical situation most closely in the region around $R_{c}$, where $t\left(R_{c}\right)=1$. However, at large values of $b$ (the impact parameter), the system may never penetrate to $R_{c}$; in that case the model should be made to match the physical situation most closely when $t$ is smallest, i.e., at the turning point, $R_{0}$.

\section{Matching at $R_{c}$}

To make the model $t(s)=(\alpha \cos \zeta s)^{-1}$ match the actual $t(s)$ as closely as possible at $R_{c}$, where $t\left(R_{c}\right)=1$, we may choose $\alpha$ and $\zeta$ such that the first and second derivatives of the model and the true $t(s)$ agree at $R_{c}$.

In the model, $t\left(s_{c}\right)=1$ implies

$$
\cos \zeta s_{c}=\alpha^{-1}
$$

and

$$
\begin{aligned}
& \left.\frac{d t}{d s}\right|_{s_{c}}=\frac{\zeta \sin \zeta s_{c}}{\alpha \cos ^{2} \zeta s_{c}}=\zeta\left(\alpha^{2}-1\right)^{1 / 2}, \\
& \left.\frac{d^{2} t}{d s^{2}}\right|_{s_{c}}=\frac{\zeta^{2}\left(1+\sin ^{2} \zeta s_{c}\right)}{\alpha \cos ^{3} \zeta s_{c}}=\zeta^{2}\left(2 \alpha^{2}-1\right) .
\end{aligned}
$$

In the actual system,

$\left.\frac{d t}{d s}\right|_{R_{c}}=\left(\frac{d t}{d R}\right)\left(\frac{d R}{d \tau}\right)\left(\frac{d \tau}{d s}\right)=\left(\frac{d t}{d R}\right)\left(\frac{v \hbar}{V_{12}}\right)$,

and similarly

$\left.\frac{d^{2} t}{d s^{2}}\right|_{R_{c}}=\left(\frac{d^{2} t}{d R^{2}}\right)\left(\frac{\hbar^{2} v^{2}}{V_{12}^{2}}\right)+\left(\frac{d t}{d R}\right)\left[\left(\frac{F \hbar^{2}}{m V_{12}^{2}}\right)-\frac{v^{2} \hbar^{2} V_{12}^{\prime}}{V_{12}^{3}}\right]$.

Here $v$ is the average nuclear velocity and $F$ is the average force, including the angular momentum repulsion; all these quantities as well as $V_{12}$ and the derivatives of $t$ with respect to $R$ are to be evaluated at $R_{c}$. Obviously some minimal knowledge of the potential curves is required to evaluate these quantities. Once (15) is evaluated, $\alpha$ and $\zeta$ follow from (14):

$$
\begin{aligned}
& \zeta=\left[\frac{d^{2} t}{d s^{2}}-2\left(\frac{d t}{d s}\right)^{2}\right]^{1 / 2}, \\
& \alpha=\left[\frac{d^{2} t / d s^{2}-(d t / d s)^{2}}{d^{2} t / d s^{2}-2(d t / d s)^{2}}\right]^{1 / 2},
\end{aligned}
$$

where all quantities are to be evaluated at $R_{c^{\circ}}$ [The 
parameters $\alpha$ and $\zeta$ must be real, and if for some system the radicands in Eq. (16) are negative, it means that the approximations leading to (16) are not valid.]

\section{Matching at $R_{0}$}

At the turning point, $d t / d s=0$. Therefore, to make the model match the actual $t(s)$ in the region of the turning point, we may choose $\alpha$ and $\zeta$ so that the value and the second derivative of the model and of the true $t(s)$ agree at $R_{0}$. For the model

$$
t(0)=\alpha^{-1},\left.\frac{d^{2} t}{d s^{2}}\right|_{s=0}=\frac{\zeta^{2}}{\alpha}
$$

and for the actual system,

$$
t(0)=\frac{V_{22}-V_{11}}{2 V_{12}},\left.\frac{d^{2} t}{d s^{2}}\right|_{s=0}=\left(\frac{d t}{d R}\right) \frac{F \hbar^{2}}{m V_{12}^{2}},
$$

where all quantities are to be evaluated at the turning point. Thus, obviously,

$$
\alpha=[t(0)]^{-1}, \quad \zeta=\left\{\left.\frac{d^{2} t / d s^{2}}{t}\right|_{s=0}\right\}^{1 / 2} .
$$

\section{Matching $t(0)$ and $S_{\infty}$}

A third way of choosing the parameters in the model is also useful for some applications. We may choose $\alpha$ and $\zeta$ such that the values of $t(0)$ and $s_{\infty}$ in the model match the values in the physical system. Thus, $\alpha$ and $\zeta$ are chosen such that

$$
\begin{aligned}
& 1 / \alpha=t(0), \\
& \frac{\pi}{2 \zeta}=s_{\infty}=\frac{1}{\hbar} \int_{R_{0}}^{\infty} V_{12} \frac{d \tau}{d R} d R .
\end{aligned}
$$

This is only useful if the integral is simple; such a case arises if the coupling is exponential,

$$
V_{12}=A e^{-\lambda R}
$$

and $R_{c}$ is sufficiently large that the forces are weak and the trajectory is essentially a straight line with constant velocity. In this case,

$$
\begin{aligned}
R^{2} & =b^{2}+v^{2} \tau^{2}, \\
s_{\infty} & =\int_{b}^{\infty} A e^{-\lambda R} \frac{R}{\hbar v\left(R^{2}-b^{2}\right)^{1 / 2}} d R \\
& =(A / \hbar v) b K_{1}(\lambda b) \\
& \simeq \frac{A}{\hbar v}\left(\frac{\pi b}{2 \lambda}\right)^{1 / 2} e^{-\lambda b}\left(1+\frac{3}{8 \lambda b}+\cdots\right) .
\end{aligned}
$$

Here $K_{1}$ is the modified Bessel function, and the last line gives its asymptotic expansion for large $\lambda b$. [In principle a somewhat better approximation to the coupling matrix element is $V_{12}=A R e^{-\lambda R}$; for this case, $s_{\infty}$ can be obtained by differentiating (22a) with respect to $\lambda$. However, for our purposes, (22b) proved satisfactory.]

It is important that we have not assumed that the radial velocity is constant, only that the trajectory is rectilinear, so the three-dimensional vector velocity is constant. The constant vector velocity approximation is often accurate, even when the constant radial velocity approximation is not. It is the neglect of radial acceleration that makes the Landau-Zener formula fail when the crossing point is close to the turning point, and likewise it makes the Demkov formula fail when $R_{c}$ is close to the turning point. In either case, the constant vector velocity approximation can often be used to obtain accurate results.

\section{A combined formula}

Some of the above results can be combined to give a still more accurate representation of the transition probability. If $R_{0}$ is less than $R_{c}$, we may calculate $\pi / \alpha \zeta$, the argument of the $\mathrm{sech}^{2}$ factor, from Eq. (16), and calculate $\pi / \zeta$, the argument of the $\sin ^{2}$ factor, from Eq. (20b). [In this way we are calculating the average transition probability from quantities that are most directly related to the behavior of $t(s)$ near the critical point, and we are calculating the phase from a quantity that is appropriately related to the entire path difference.] The resulting formula is

$$
\begin{aligned}
P= & \operatorname{sech}^{2}\left\{\frac{\pi}{\left[\left(d^{2} t / d s^{2}\right)-(d t / d s)^{2}\right]^{1 / 2}}\right\} \\
& \times \sin ^{2}\left\{\frac{2}{\hbar} \int_{0}^{\infty} V_{12}\left(\tau^{\prime}\right) d \tau^{\prime}\right\}, \quad R_{0}<R_{c},
\end{aligned}
$$

where the derivatives of $t$ are evaluated at $R_{c^{\circ}}$

For $R_{0}$ greater than $R_{c}$, the appropriate argument for the $\operatorname{sech}^{2}$ factor would be obtained from Eq. (19), so that

$$
\begin{aligned}
P= & \operatorname{sech}^{2}\left\{\frac{\pi t^{3 / 2}}{\left(d^{2} t / d s^{2}\right)^{1 / 2}}\right\} \\
& \times \sin ^{2}\left\{\frac{2}{\hbar} \int_{0}^{\infty} V_{12}\left(\tau^{\prime}\right) d \tau^{\prime}\right\},
\end{aligned}
$$

where $t$ and its second derivative are to be evaluated at the turning point.

If, in addition, the coupling is approximated by an exponential function (21), and the trajectory is approximated by a straight line, then the argument of the $\sin ^{2}$ factor is given by (22b). In Sec. IV A the resulting formula is compared with exact numerically determined transition probabilities, and it is found to be quite accurate, especially for $R_{0}<R_{c^{\circ}}$ 


\section{Qualitative interpretation}

Since the physical significance of the above formulas may be less than completely obvious, it is useful to write some of them in a different form. Let us define the distance $r_{0}$ over which $t(R)$ changes by an amount that is comparable to its own value,

$$
r_{0}=\left(t / \frac{d t}{d R}\right)_{R_{0}}
$$

and let us define the time $\tau_{0}$ as the time required for the nuclei to the move a radial distance $r_{0}$ from the turning point under the action of the constant force $F$ :

$$
\tau_{0}=\left(2 M r_{0} / F\right)^{1 / 2} \text {. }
$$

Thus $\tau_{0}$ is the time required for $t$ to change by an amount that is comparable to its value. Using this notation, and defining $\Delta=\left(V_{22}-V_{11}\right)_{R_{0}}$, we find that (18) and (19) imply that

$$
P=\operatorname{sech}^{2}\left[\left(\frac{\pi^{2}}{8}\right)^{1 / 2} \frac{\Delta \tau_{0}}{\hbar}\right] \times \sin ^{2}\left[\left(\frac{\pi^{2}}{8}\right)^{1 / 2} \frac{2 V_{12} \tau_{0}}{\hbar}\right] .
$$

The interpretation of this formula is more clear. The first factor says that transitions are unlikely to take place if the collision is so slow that the uncertainty principle can distinguish between the two states, $i_{\circ} e_{\circ}$, there are few transitions if

$$
\Delta \tau_{0} \gtrsim \hbar \text { 。 }
$$

The second factor says that transitions are unlikely unless the coupling has sufficient strength that it can act in the time $\tau_{0}$; i.e., there are few transitions if

$$
2 V_{12} \tau_{0} \ll \hbar \text {. }
$$

If the coupling is very strong, the system can jump back and forth between the two states, and the resulting overall transition probability is oscillatory.

While (24) is easy to understand, and useful for order-of-magnitude estimates, it is not suitable for accurate quantitative calculations. On the other hand, the accurate formulas, (15) and (16), or (20)-(22), or (23), are not so easy to interpret, though they do have essentially the same meaning as (24). It is best to simply repeat that they provide various ways of approximating the physical $t(s)$ (10) by the model (11), and that a good approximation to $t(s)$ is sufficient to obtain a good approximation to the transition probability.

\section{TRANSITION PROBABILITY AND TOTAL CROSS SECTION}

In this section we show how the above formulas can be used in model calculations and in actual physical systems to obtain the transition probability as a function of impact parameter and velocity, and to obtain the total cross section as a function of velocity. In the calculations of this section, we assume that we are given two potential curves, $V_{11}$ and $V_{22}$, such that their difference, $V_{22}-V_{11}$, is small and slowly varying. The coupling is an exponential function of distance, (21). We assume that $R_{c}$ is sufficiently large that the forces are weak, and the trajectory is essentially rectilinear.

\section{A. Transition probability$$
\text { 1. } P \text { vs } b \text { at fixed } v_{0}
$$

Let us first consider the behavior of the transition probability as a function of impact parameter. Suppose the potential curves are such that, in atomic units,

$$
\begin{aligned}
& \Delta \equiv V_{22}\left(R_{c}\right)-V_{11}\left(R_{c}\right)=0.02, \\
& \lambda=0.3,
\end{aligned}
$$

and the asymptotic velocity is

$$
v_{0}=0.1045 \text {. }
$$

The transition probability is obtained from (13). If we use the method of Sec. III B 3 above to determine the parameters $\alpha$ and $\zeta$, then from (13), (20a), (21), and (22)

$$
\begin{aligned}
P= & \operatorname{sech}^{2}\left[\frac{\Delta}{\hbar v_{0}}\left(\frac{\pi b}{2 \lambda}\right)^{1 / 2}\left(1+\frac{3}{8 \lambda b}+\cdots\right)\right] \\
& \times \sin ^{2}\left[\frac{2 A}{\hbar v_{0}}\left(\frac{\pi b}{2 \lambda}\right)^{1 / 2} e^{-\lambda b}\left(1+\frac{3}{8 \lambda b}+\cdots\right)\right] .
\end{aligned}
$$

Equation (28) is essentially the same as the RappFrancis approximation, ${ }^{9}$ which has been used for many years to study nonresonant charge exchange.

Let us first determine the accuracy of the truncated asymptotic series expansion (22b) for the Bessel function appearing in (22a). If $A$ is large, the transition probability may be rapidly oscillating; therefore we show in Fig. 2 only the enve-

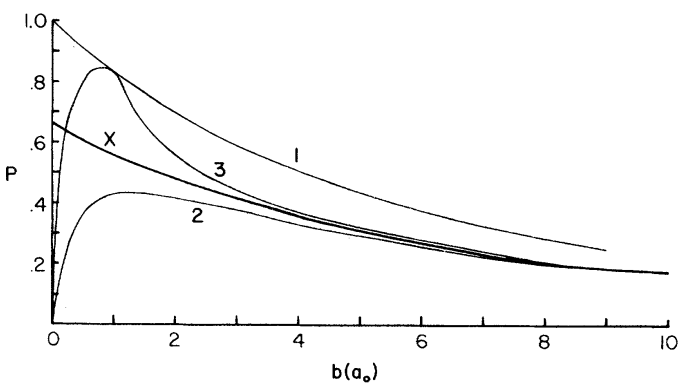

FIG. 2. Envelope of the transition probability vs impact parameter $b$. Exact Bessel function form $(x)$ is compared with one-term, two-term, and three-term asymptotic approximation. 
lope of $P$ (the sech factor) as a function of $b$ for the parameters listed in Eq. (27). We compare the various orders in the asymptotic series with the full Bessel function of (22a). It is clear that the first two terms in the asymptotic expansion agree with the full Bessel function except for $b<3 a_{0}$. If $R_{c}$ is large (for $A=1, R_{c} \simeq 17 a_{0}$ in this case), then the error in $P$ at small $b$ will have only a small effect on the calculated total cross section, and the two term asymptotic expansion will be adequate.

In Fig. 3(a) is shown the full transition probability (28) for the parameters (27) and

$$
A=1 \text {. }
$$

This formula is compared to the result of an exact numerical close coupled calculation by Olson for a comparable situation. At this fairly low velocity, the magnitude of the transition probability is somewhat small, but the phase of the oscillations is surprisingly accurate. More commonly, one finds the magnitude to be in better agreement and the phase to be in worse agreement: interference patterns are very sensitive to details of the potential curves, and general models like this one often do
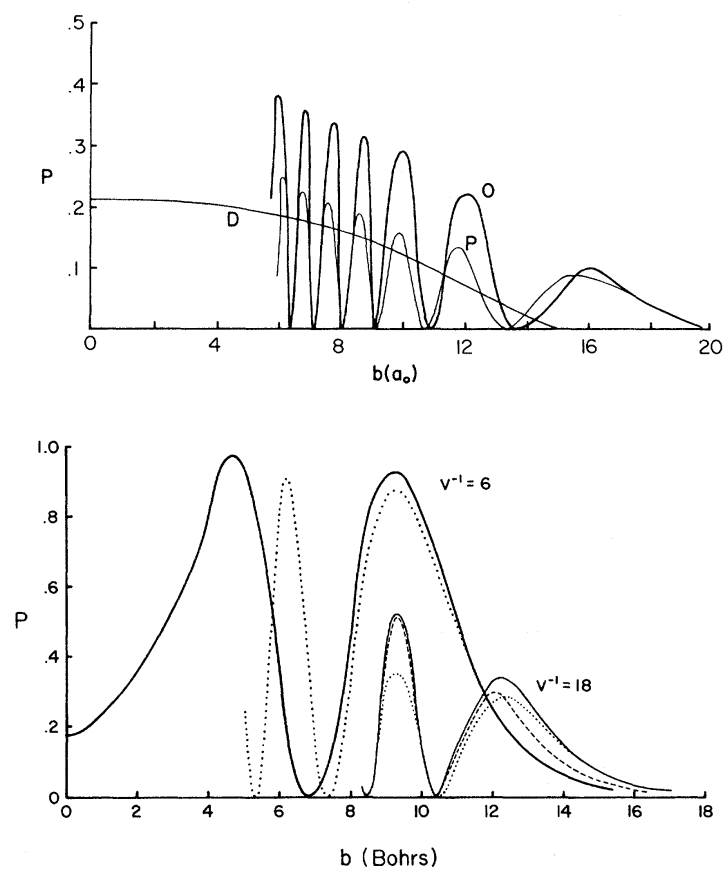

FIG. 3. Transition probability vs impact parameter at fixed velocity; comparison of Eq. (28) with exact results. (a) Curve $O$, an exact calculation by Olson. Curve $D$, Demkov formula for the same case. Curve P, present formula (28) with $A=1.0, \lambda=0.3, \Delta=0.02$. (b) Solid curve, exact (two state) calculation of Melius and Goddard. Dotted curve, present formula (28). Dashed curve, combined formula (23a), (23b), (22b). not reproduce them accurately.

The present results are also compared to the result of the Demkov formula. ${ }^{2}$ The Demkov formula accurately reproduces the average of the transition probability at small $b$, but it fails to reproduce the transition probability in the very important region where $b \simeq R_{c}$. Because of this, as Olson has pointed out, the Demkov formula significantly underestimates the total cross section. The present formula is quite good in the region $b \simeq R_{c}$, and, as we shall see, it gives quite accurate total cross sections.

Melius and Goddard ${ }^{8}$ have made an excellent and very thorough study of charge exchange in the $(\mathrm{Li}-\mathrm{Na})^{+}$system, and their numerical results provide another test of our formulas. In Fig. 3(b), the solid line shows their numerically determined transition probability as a function of $b$ at two different velocities. Using the method discussed below [see Eq. (36)], we have transformed their adiabatic potential curves into the diabatic representation, and used the resulting potential parameters to calculate the transition probability. The dotted line shows the result of Eq. (28) (the "RappFrancis" form); it is seen to be in quite good agreement with the exact numerical result except that it is rather small at small $v$ and small $b$. The dashed line shows the result of the combined formula (23a), (23b), and (22b) at $v^{-1}=18$. We see in Fig. $3(\mathrm{~b})$ that although for $b>12$, the resulting transition probability $(23 \mathrm{~b})$ is slightly too small, at smaller $b(8<b<10)$ it is indistinguishable on the graph from the numerical result. The only severe discrepancy between the formulas and the exact numerical results is in the phase of the oscillations at small $b$. This phase can be improved, for example by using the exact (numerically determined) value for $\int V_{12} d \tau$, but this phase is usually not important for total cross section calculations, and the loss of simplicity tends to cancel any gain in accuracy.

\section{P vs $v_{0}$ at fixed $b$}

The overall shape of the total cross section as a function of velocity is determined by the velocity dependence of the transition probability. Therefore, it is of interest to consider $P$ as a function of velocity at fixed impact parameter. Figure 4 shows the transition probability from Eq. (28), with the parameters (27a) and (29). The complete transition probability is oscillatory at low velocities, and the envelope (the $\operatorname{sech}^{2}$ factor) goes approximately as $v_{0}^{4}$ as $v_{0} \rightarrow 0$. As the envelope levels off, the $\sin ^{2}$ factor becomes small, cutting off $P$ at high velocities.

It is interesting to compare this result with the 


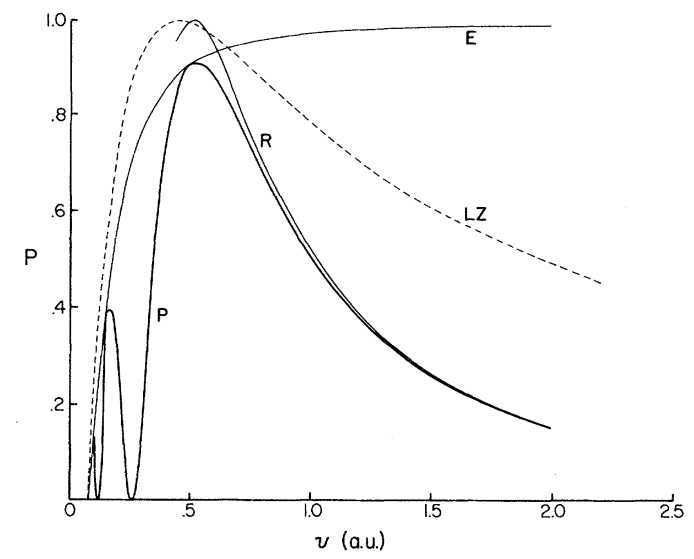

FIG. 4. Transition probability vs velocity at fixed impact parameter. Curve P, Eq. (28). Curve E, envelope of Eq. (28) ( $\operatorname{sech}^{2}$ factor). Curve R, $\sin ^{2}$ factor. Curve LZ, a comparable Landau-Zener transition probability.

result of the Landau-Zener-Stueckelberg formula, which applies to systems with a curve crossing:

$$
P=4 e^{-w / v_{0}}\left(1-e^{-w / v_{0}}\right) \sin ^{2} \chi,
$$

where $w$ is a constant related to the potential parameters at the crossing and $\chi$ represents the phase difference on the two potential curves. We took $w \simeq 0.33$ and plotted the envelope in Fig. 4 (omitting the $\sin ^{2}$ factor). The result is quite similar to the result of Eq. (28). Inclusion of the $\sin ^{2}$ factor would also introduce oscillations at low velocities, and the $L Z$ result is known to be too large at high velocities (i.e. larger than the exact transition probability for the curve crossing). It follows that although crossings and noncrossings are in principle very different, the transition probabilities can sometimes be quite similar.

\section{B. Behavior of the total cross section}

Having obtained an analytic formula, (28), for the transition probability as a function of impact parameter and velocity, it is a simple matter to integrate it numerically to obtain the total inelastic cross section,

$$
Q=2 \pi \int_{0}^{\infty} P\left(b, v_{0}\right) b d b
$$

The result is a function of $\Delta / \lambda^{1 / 2} v_{0}, A / \lambda^{1 / 2} v_{0}$, and $\lambda$, or, more simply, a function of all three constants, $\Delta, \lambda, A$ as well as $v_{0}$. However, it can be argued that $Q$ is primarily a function of just one variable, $\lambda v_{0} \hbar / \Delta$. We mentioned earlier that the transition probability depends mainly upon the behavior of $t(s)$ where $t \simeq 1$, and clearly the most important factor is the rate of change of $t$ in this region. From (15a), and our assumptions about

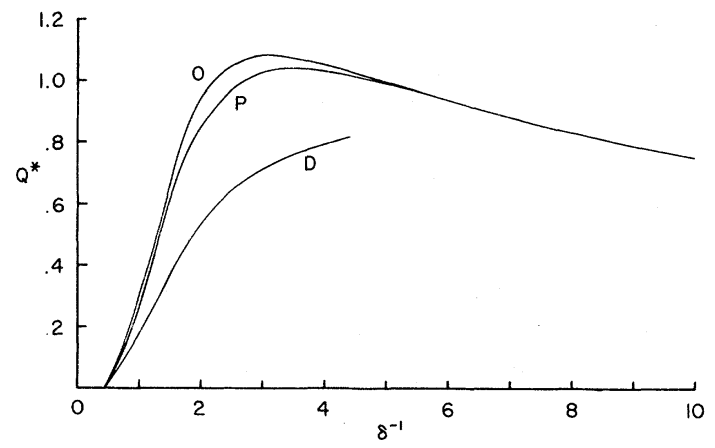

FIG. 5. Total reduced cross section vs reduced velocity. Curve O, Olson's universal curve. Curve P, present result for a comparable case $(A=1.0, \lambda=0.3$, $\Delta=0.02)$.

the potentials given at the beginning of this section, we have

$$
\left(\frac{d t}{d s}\right)_{R_{c}}=\frac{2 \lambda \hbar v_{0}}{\Delta} .
$$

Accordingly, it is reasonable to expect that the cross section will depend primarily upon the variable $\delta^{-1}=2 \lambda \hbar v_{0} / \pi \Delta$ and that it will be less sensitive to $A$, or to $\lambda$ or $\Delta$ separately. This conclusion also follows from Demkov's formula, ${ }^{2}$ and it is the basis of Olson's description ${ }^{6}$ of the total cross section as a universal function of $\delta^{-1}$.

We have calculated the total cross section from (31) and (28) for a range of the parameters, $\Delta, \lambda$, $A$, and $v_{0}$, and the results are shown in Figs. 5 and 6 . Figure 5 presents the reduced total cross

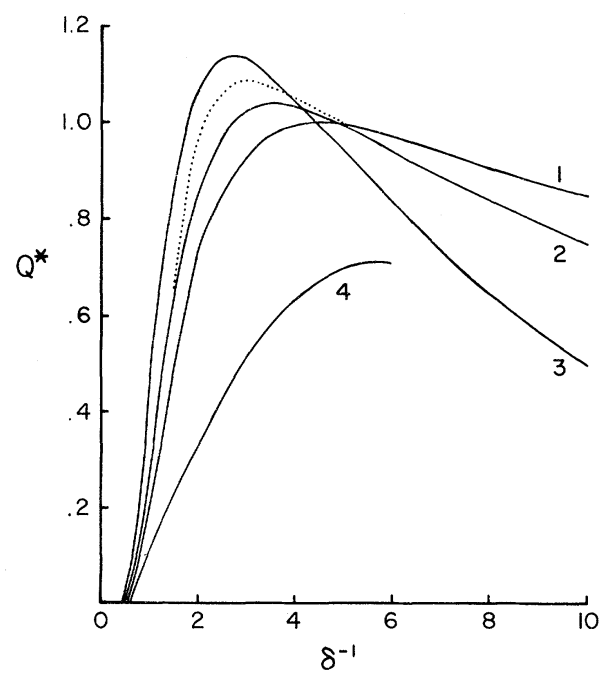

FIG. 6. Total reduced cross section vs reduced velocity with varying parameters. Curve $1, A / \Delta=200$, $\lambda=0.3$. Curve 2, $A / \Delta=50, \lambda=0.3$. Curve 3, $A / \Delta=12.5$, $\lambda=0.3$. Curve $4, A / \Delta=50, \lambda=0.1$. Dotted curve: Universal cross section of Olson. 
section, $Q^{*} \equiv Q /\left(\frac{1}{2} \pi R_{c}^{2}\right)$, for the same model parameters as considered above [Eqs. (27a) and (29)]. The values of these parameters are very similar to those used by Olson when he obtained his universal curve by direct numerical integration of the coupled Schrödinger equations. Very good agreement is found between the present calculation, with its simple analytic formula for $P$, and the numerical calculations of Olson. For comparison, the result of the Demkov formula is also shown; it seriously underestimates the total cross section because of its failure in the turning point region.

Because the present model is very flexible, we can vary the parameters independently, and test the universality of Olson's proposed universal curve. It is convenient to rewrite Eq. (28) in the form

$$
\begin{aligned}
P= & \operatorname{sech}^{2}\left[\delta(2 \lambda b)^{1 / 2}(1+3 / 8 \lambda b)\right] \\
& \times \sin ^{2}\left[(2 A / \Delta) \delta(2 \lambda b)^{1 / 2} e^{-\lambda b}(1+3 / 8 \lambda b)\right]
\end{aligned}
$$

and to think of the cross section as a function of $\delta$, $\Delta / A$, and $\lambda$. These results are shown in Fig. 6 . When $\Delta / A$ is increased, the peak in $Q^{*}$ vs $\delta^{-1}$ becomes more pronounced, and the cross section decreases more steeply at large $\delta^{-1}$. This is not surprising, because it is the $\sin ^{2}$ factor that cuts off $P\left(v_{0}, b\right)$ at either large $b$ or large $v_{0}$, and the argument of the $\sin ^{2}$ is proportional to $A / \Delta$. When $\lambda$ is varied, an increase in its value by a factor of 3 does not visibly change the curve; however, decreasing $\lambda$ (to a rather nonphysical value) does have an effect.

It is also of interest to compare the calculated total cross section with cross sections for resonant collisions. At high velocities, the system does not "feel" the two potentials, but only an average potential and a coupling. For example, in Eq. (2), as $v_{0}$ gets large, $\int_{0}^{\tau}\left(V_{22}-V_{11}\right) d \tau$ gets small, and the exponential factor approaches unity; therefore, the transition probability is determined mainly by $V_{12}(\tau)$. The resonant limit to the transition probability and the cross section is obtained by setting $\Delta=0$ in Eq. (28). In Fig. 7 the calculated cross section for collisions involving small energy defects is compared to the resonant limit at high velocities. This is very pleasing, because it is not easy to obtain formulas for transition probabilities that are valid over a broad range of velocities - for example, for the curvecrossing problem, there is no single analytic formula that is valid for general coupling strengths at both high and low velocities. It is also seen in Fig. 7 that the sharp drop in the total cross section as the velocity decreases reflects the actual nonresonant nature of this process.

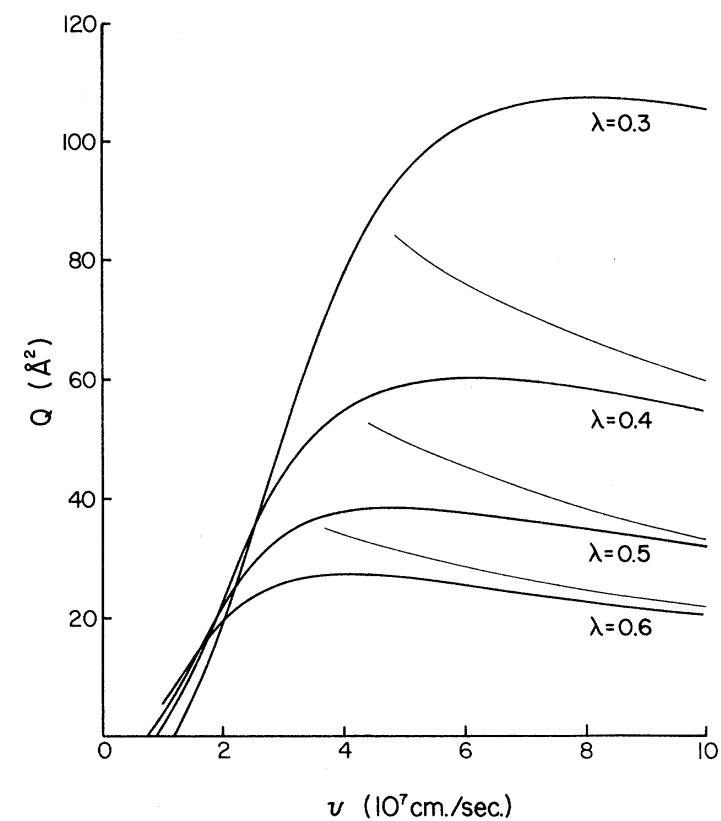

FIG. 7. Total cross sections vs velocity, compared to resonant limit. Each has $A / \Delta=50$, with varying $\lambda$.

\section{Charge exchange in $\mathrm{Li}^{+}+\mathrm{Na}$}

We now apply these results to an actual physical situation, and compare the predicted cross sections with experiments. The total cross section for charge exchange in alkali metals has been measured by Daley and Perel, ${ }^{10}$ and a very detailed and careful theoretical study was carried out by Melius and Goddard. ${ }^{8}$ More approximate calculations were made by $\mathrm{Olson}^{6}$ and by Bottcher and Oppenheimer. ${ }^{7}$ Olson $^{6}$ made some simple analytic approximations to the potential curves in the diabatic representation, and calculated the cross section by using his universal curve. Bottcher and Oppenheimer ${ }^{7}$ and Melius and Goddard ${ }^{8}$ made approximate calculations of the potential curves in the adiabatic representation; while their potentials are quite similar, their coupling matrix elements differ substantially. Bottcher and Oppenheimer then calculated the total charge exchange cross section by first-order perturbation theory, while Melius and Goddard calculated it by direct numerical integration of the classical trajectory equations. There are significant discrepancies between the various approaches, and in this section we develop some of the relations between them, and carry out calculations by the methods developed above.

\section{Diabatic and adiabatic representations}

Melius and Goddard ${ }^{8}$ and Bottcher and Oppenheimer ${ }^{7}$ have made approximate calculations of 
the potential curves and the $d / d R$ coupling matrix element in the adiabatic representation. In order to use the formalism of this paper, these calculations have to be transformed into the diabatic representation。

F. T. Smith ${ }^{13}$ has suggested that the diabatic representations can be defined for the two state problem as those representations in which the nuclear momentum operator $(\hbar / i) d / d R$ does not couple the two electronic states; i.e.,

$$
P_{12}^{D}=\left\langle\Phi_{1}^{D}(r ; R)\left|\frac{\hbar}{i} \frac{d}{d R}\right| \Phi_{2}^{D}(r ; R)\right\rangle,
$$

where the scalar product involves an integration over electronic but not nuclear coordinates. A second condition is also required to make the definition unique:

$$
\lim _{R \rightarrow \infty} V_{12}(R)=0 \text {. }
$$

(The resulting states are not simply eigenfunctions for the separated atoms, ${ }^{14}$ because other matrix elements of $P$ have not been made to vanish: only $P_{12}^{D}=0$.)

With this definition, the formulas relating the diabatic and adiabatic representations have been given in Refs. 2(b) and 3(a). They are connected by a matrix $W$ such that

$$
\begin{aligned}
& {\left[\begin{array}{l}
\phi_{1}^{A}(r ; R) \\
\phi_{2}^{A}(r ; R)
\end{array}\right]=\underline{W}\left[\begin{array}{c}
\phi_{1}^{D}(r ; R) \\
\phi_{2}^{D}(r ; R)
\end{array}\right],} \\
& \underline{W}=\left[\begin{array}{rr}
\cos \theta & -\sin \theta \\
\sin \theta & \cos \theta
\end{array}\right] .
\end{aligned}
$$

The angle $\theta(R)$ is defined such that the potential matrix is diagonal,

$$
\underline{W} \underline{V} \underline{W}^{-1}=\underline{\epsilon} \text { (diagonal), }
$$

and from the above it is easy to show that

$$
\begin{aligned}
P_{12}^{A} & =\left\langle\phi_{1}^{A}(r ; R)\left|\frac{\hbar}{i} \frac{d}{d R}\right| \phi_{2}^{A}(r ; R)\right\rangle \\
& =\frac{\hbar}{i} \frac{d \theta}{d R} .
\end{aligned}
$$

In the adiabatic representation, $\epsilon_{1}(R), \epsilon_{2}(R)$, and $P_{12}(R)$ must be given. To obtain the diabatic representation, $\theta(R)$ must be calculated by integration of (35); then $V(R)$ is obtained from (34): $V=W^{-1} \in W$. The angle $\theta \overline{(R)}$ is determined only to within an arbitrary additive constant; this must be taken such that $\lim _{R \rightarrow \infty} V_{12}(R)=0$, or equivalently $\lim _{R \rightarrow \infty} \theta(R)=0$.

\section{Calculation of total cross section}

We have applied the above formulas to obtain the $2 \times 2$ potential matrix in the diabatic representa- tion. The resulting potential matrix elements are well approximated by the forms

$$
\begin{aligned}
& V_{11}=1.152 e^{-0.7253 R} \\
& V_{22}=V_{11}+0.00925, \\
& V_{12}=0.8493 e^{-0.4034 R} .
\end{aligned}
$$

(This is in essential agreement with Melius and Goddard.) Therefore the appropriate values of the parameters are

$$
A=0.8493, \lambda=0.4034 \text {. }
$$

Using these parameters in our Eqs. (28) and (31), we obtain a total cross section for $(\mathrm{Li}-\mathrm{Na})^{+}$charge exchange which is shown in Fig. 8. The agreement with experiment is very good.

Bottcher and Oppenheimer ${ }^{7}$ have also calculated adiabatic potentials and coupling matrix elements for this system. While their potential curves are essentially the same as those of Melius and Goddard, ${ }^{8}$ their coupling matrix element is quite different. Bottcher and Oppenheimer have calculated the total cross section by a first-order approximation, and their result is about $50 \%$ too large. They have interpreted their results qualitatively in terms of curve-crossing theory, asserting that there is a crossing at $10.8 a_{0}$.

However, when their curves are transformed into the diabatic representation, no such crossing appears. The diagonal matrix elements run approximately parallel in to about $12 a_{0}$, where they rapidly diverge from each other. The coupling matrix element is slowly varying in this region. This behavior is completely different from that predicted by Olson and Melius and Goddard, and our model cannot be used to obtain the cross section. The discrepancy between the calculations of Bottcher and Oppenheimer and the experimental results may be due partly to the first-order cal-

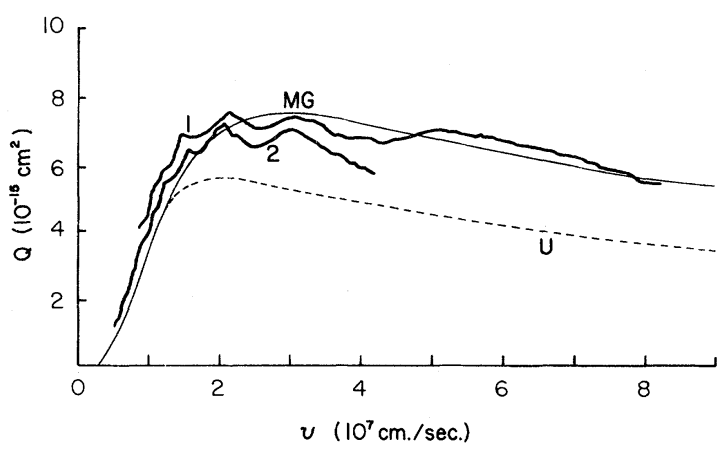

FIG. 8. Total cross section vs velocity for $(\mathrm{Li}-\mathrm{Na})^{+}$ charge exchange. Curve MG: present result with Melius and Goddard potentials. Curve U: universal curve; from Olsen's approximate potentials. Heavy wiggly lines, experimental results of Daley and Perel. 
culation they employed, and partly to the difference between their potentials and those of Melius and Goddard.

\section{Discussion}

The Melius and Goddard calculation of the total cross section should be the best theoretical prediction, because it is based upon a direct calculation of the potential curves and an exact numerical solution to the classical trajectory equations. One of our calculations uses their potentials transformed into the diabatic representation and fit to our model. It is seen that the resulting prediction is in good agreement with the results of Melius and Goddard; this shows that the model is an accurate representation of the actual physical system. It is noted, however, that the oscillations in the total cross section are not reproduced in our model. It is believed that these oscillations result from a point of stationary phase related to a maximum in $\epsilon_{1}(R)-\epsilon_{2}(R)$. The present model could generate such oscillations if an improved approximation were used for the argument of the $\sin ^{2}$ factor.

Olson's calculation is less accurate than that of Melius and Goddard partly because he used a simpler and less accurate approximation to the potential curves, and partly because his universal curve cannot in general be accurate as an exact numerical calculation. We recalculated Olson's universal curve for the $(\mathrm{Li}-\mathrm{Na})^{+}$system using the Melius and Goddard approximation to the potentials, and we found it to be very close to the result of our calculation using the generalized RosenZener model. Apparently this is one of the cases for which the universal curve is quite accurate, and the principal cause of the earlier discrepancy was Olson's simpler approximation to the potential curves.

\section{SUMMARY}

We have shown that the formalism developed in Ref. 4(b), and particularly the formulation of twostate problems in terms of the dimensionless function $t(s)$, provides a useful exactly solvable model for noncrossing interactions. Various approximations for the parameters in the model lead to the Rapp-Francis formula, or to other formulas for the transition probability, the most accurate of which is (23a). The Rapp-Francis form was then used to study total cross sections for charge exchange in the $(\mathrm{Li}-\mathrm{Na})^{*}$ system.

\section{APPENDIX: CALCULATION OF THE $S$ MATRIX}

The object is to solve Eqs. (9), with the model $t(s)$ of Eq. (11), to obtain the $S$ matrix of Eq. (3) and the $G$ matrix of Eq. (4) and (5).

Equation (9) may be written as a second-order differential equation for $c_{1}$ :

$$
\frac{d^{2} c_{1}}{d s^{2}}+2 i t \frac{d c_{1}}{d s}+c_{1}=0 \text {. }
$$

By making the substitution

$$
z=\frac{1}{2} \sin \zeta s+\frac{1}{2},
$$

we obtain the standard form of the hypergeometric equation,

$z(1-z) \frac{d^{2} c_{2}}{d z^{2}}+[c-(a+b+1) z] \frac{d c_{2}}{d z}-a b c_{2}$,

where

$$
\begin{aligned}
& a=-b=1 / \zeta, \\
& c=\frac{1}{2}-i / \alpha \zeta \equiv \frac{1}{2}-i \gamma .
\end{aligned}
$$

The variable $z$ spans the range $[0,1]$ as time goes from $-\infty$ to $\infty$.

Two linearly independent solutions are

$$
\begin{aligned}
c_{2}= & A F(a, b ; c ; z) \\
& +B z^{1-c} F(a+1-c, b+1-c ; 2-c ; z),
\end{aligned}
$$

and the coefficients $A$ and $B$ can be obtained from the boundary conditions

$$
\begin{aligned}
& c_{1}(z=0)=1, \\
& c_{2}(z=0)=0 .
\end{aligned}
$$

The second of these implies that $A=0$.

The value of $B$ is obtained by using (9) in the form

$$
c_{1}=\exp \left[-2 i \int_{0}^{s} t\left(s^{\prime}\right) d s^{\prime}\right] i \frac{d c_{2}}{d s},
$$

and using Eq. (15.2.4) of Abramowitz and Stegun. ${ }^{15}$ The boundary condition (A7a) then implies

$$
\begin{aligned}
B & =\frac{-i}{(1-c) \zeta} \lim _{z \rightarrow 0}\left\{z^{i \gamma} \exp \left[2 i \int_{0}^{\pi / 2 \zeta}(\alpha \cos \zeta s)^{-1} d s\right]\right\} \\
& =-\frac{i a}{c^{*}}=\frac{-i}{\zeta(1 / 2+i / \alpha \zeta)} .
\end{aligned}
$$

The transition probability is given by

$$
P=\left|c_{2}(z=1)\right|^{2} \text {, }
$$

and, by using ${ }^{15}(15.1 .20)$,

$$
c_{2}(z=1)=B z^{1-c} \frac{\Gamma(2-c) \Gamma(c)}{\Gamma(1-a) \Gamma(1+a)} .
$$

The $\Gamma$ functions can be simplified by using the well-known reflection formula ${ }^{15}(6.1 .17)$, with the result

$$
\begin{aligned}
& c_{2}(z=1)=(-i) \operatorname{sech}(\pi / \alpha \zeta) \sin (\pi / \zeta), \\
& P=\operatorname{sech}^{2}(\pi / \alpha \zeta) \sin ^{2}(\pi / \zeta) .
\end{aligned}
$$


In order to obtain the $G$ matrix, which is used in the differential cross-section calculations, it is necessary to evaluate $c_{1}\left(z=\frac{1}{2}\right)$ and $c_{2}\left(z=\frac{1}{2}\right)$. To obtain $c_{2}$, it is only necessary to apply ${ }^{15}(15.1 .25)$, and one obtains

$$
\begin{aligned}
c_{2}\left(z=\frac{1}{2}\right)=- & i\left(\frac{1}{2} \pi\right)^{1 / 2}\left(\frac{1}{2}\right)^{i \gamma} \Gamma\left(\frac{1}{2}+i \gamma\right) \\
\times & \left\{\left[\Gamma\left(\frac{1}{2}(a+1-c)\right) \Gamma\left(\frac{1}{2}+\frac{1}{2}(b+1-c)\right)\right]^{-1}\right. \\
& \left.-\left[\Gamma\left(\frac{1}{2}+\frac{1}{2}(a+1-c)\right) \Gamma\left(\frac{1}{2}(b+1-c)\right)\right]^{-1}\right\} .
\end{aligned}
$$

To obtain $c_{1}$, we must first apply the Gauss relation for contiguous functions ${ }^{15}(15.2 .20)$, which in the present case takes the form,

$$
\begin{aligned}
F(a+1-c, & \left.b+1-c ; 1-c ; \frac{1}{2}\right) \\
= & 2 F\left(a-c, b+1-c ; 1-c ; \frac{1}{2}\right) \\
& -[a /(1-c)] F\left(a+1-c, b+1-c ; 2-c ; \frac{1}{2}\right) .
\end{aligned}
$$

Then it is possible to apply ${ }^{15}(15.1 .24)$ to the first term, and $\mathrm{d}^{15}(15.1 .25)$ to the second; there is a partial cancellation leading to

$$
\begin{aligned}
c_{1}\left(z=\frac{1}{2}\right)=\left(\frac{1}{2} \pi\right)^{1 / 2}\left(\frac{1}{2}\right)^{i \gamma} \Gamma(1-c) \\
\times\left\{\left[\Gamma\left(\frac{1}{2}(a+1-c)\right) \Gamma\left(\frac{1}{2}+\frac{1}{2}(b+1-c)\right)\right]^{-1}\right. \\
\\
\left.+\left[\Gamma\left(\frac{1}{2}+\frac{1}{2}(a+1-c)\right) \Gamma\left(\frac{1}{2}(b+1-c)\right)\right]^{-1}\right\} .
\end{aligned}
$$

The expressions (A12) and (A13) evidently represent $G_{21}^{-}$and $G_{11}^{-}$respectively. The $G_{+}$matrix is the complex conjugate of $G_{-}$.

It should be noted that only the transition probability (A11) is needed for calculations of the total cross section. The more complicated forms (A12) and (A13) are useful for differential cross sections, but even there they are not absolutely essential.
*Based in part upon an Honors Thesis submitted by T. R. D. for a B. S. in Physics, College of William and Mary, 1974.

${ }^{1}$ N. Rosen and C. Zener, Phys. Rev. 40, 502 (1932).

${ }^{2}$ Yu N. Demkov, Zh. Eksp. Teor. Fiz. $\underline{45}, 195$ (1963) [Sov. Phys.-JETP 18, 138 (1964)].

${ }^{3}$ (a) J. B. Delos, W. R. Thorson, and S. K. Knudson, Phys. Rev. A 6 , 709 (1972); (b) J. B. Delos and W. R. Thorson, Phys. Rev. A 6 , 720 (1972).

${ }^{4}$ (a) W. R. Thorson, J. B. Delos, and S. A. Boorstein, Phys. Rev. A 4, 1052 (1971); (b) J. B. Delos and W. R. Thorson, Phys. Rev. A $\underline{6}, 728$ (1972); Phys. Rev. Lett. 28, 647 (1972).

5 (a) J. B. Delos, Phys. Rev. A $\underline{9}, 1626$ (1974); (b) S. M. Bobbio, L. D. Doverspike, and R. L. Champion, Phys. Rev. A ㄱ, 526 (1973).

${ }^{6}$ R. E. Olson, Phys. Rev. A 6 , 1822 (1972).

${ }^{7}$ C. Bottcher and M. Oppenheimer, J. Phys. B $\underline{5}, 492$
(1972).

${ }^{8}$ C. F. Melius and W. A. Goddard, III, Chem. Phys. Lett. 15, 524 (1972); Phys. Rev. Lett. 29, 975 (1972); Phys. Rev. A 10, 1541 (1974).

${ }^{9}$ D. Rapp and W. E. Francis, J. Chem. Phys. 37, 2631 (1962), Eq. (51).

${ }^{10}$ H. L. Daley and J. Perel, in Proceedings of the Sixth International Conference on the Physics of Electronic and Atomic Collisions, Cambridge (MIT Press,

Cambridge, Mass., 1969), p. 51.

${ }^{11}$ H. Nakamura, Mol. Phys. 25, 577 (1973).

${ }^{12}$ D. S. F. Crothers, J. Phys. B $\underline{6}, 1418$ (1973).

${ }^{13}$ F. T. Smith, Phys. Rev. 179,111 (1969).

${ }^{14} \mathrm{H}$. Gabriel and K. Taulbjerg, Phys. Rev. A $\underline{10}, 741$ (1974).

${ }^{15} \mathrm{M}$. Abramowitz and I. A. Stegun, Handbook of Mathematical Functions (U.S. GPO, Washington, D.C., 1970). 